\title{
Political and Social Consequences of Qualification Mismatches: A bounding approach to status inconsistency
}

\author{
Jonas Wiedner ${ }^{1}$ \\ WZB Berlin Social Science Center \\ wiedner@wzb.eu
}

July $24^{\text {th }} 2020$

\begin{abstract}
A significant number of employees work in jobs that do not match their level of formal education. Status inconsistency theory (SIT) argues that mismatches result in stress, political alienation, and social withdrawal. As the number of mismatched workers rises in many countries, status inconsistency may pose a threat to social cohesion and political moderation there. However, the existing evidence on the social and political consequences of mismatch is neither conclusive nor convincing. Previous SIT scholarship does not fully appreciate two identification problems: Selection bias and the perfect collinearity among the effects of occupation, education, and mismatch. These issues lead to contradictory conclusions, as different methodological fixes are proposed and employed. I review these methods for their theoretical content and show that they generally do not answer the purported research question. To address these problems, I build on recent advances in the modelling of age, period and cohort effects. My approach is based on relatively weak, transparent assumptions that are grounded in sociological theory to partially identify mismatch effects and estimate bounds on effect sizes. The empirical analysis draws on comparable large-scale survey data from the United Kingdom (UKLHS) and Germany (GSOEP). Cross-sectional and panel fixed-effects models show strong mismatch effects on work-related identities, satisfaction, and wages. Contra the SIT hypothesis, I find no evidence that mismatch effects spill over into the political domain. My results suggest that the effects of mismatches do not arise from cognitive dissonance, as theorized by SIT, but from an expectation formation mechanism. Despite large institutional differences, the results are very similar across countries.
\end{abstract}

\footnotetext{
${ }^{1}$ This paper is based on research I undertook as a doctoral student at the Institute for Sociology and Social Research of the University of Cologne. I thank Marita Jacob and Merlin Schaeffer for helpful comments on earlier versions of this manuscript.
} 


\section{Introduction}

Many employees work in occupations for which they have not been trained (Rohrbach-Schmidt and Tiemann 2016; Sloane, Battu, and Seaman 1999; Vaisey 2006). This paper investigates consequences of such qualification-job mismatches for political and social attitudes and behaviors of workers who are vertically mismatched (i.e. workers who are under- or overqualified for the occupations they work in). Social scientists have asked for more than half a century, whether such status-inconsistent employment situations lead to stress, poor health, dissatisfaction, social withdrawal, opposition to achievement ideology, political alienation, and in the last consequence to societal instability and unrest (Blocker and Riedesel 1978; Burris 1983; Goffman 1957; Hope 1975; Lenski 1954). Empirical research has generally produced highly mixed results, but more recent studies find an association between mismatches, in particular overqualification, and measures of these outcomes (Vaisey 2006; Zhang 2008; Zhu and Chen 2016). Given increasing rates of overqualification in countries like the United Kingdom and the United States, these results are clearly worrying (Felstead et al. 2007; Horowitz 2018; Vaisey 2006).

However, existing empirical work suffers from two major shortcomings that severely limit our understanding of the effects of mismatches. First, virtually all studies that investigate the effects of mismatches use cross-sectional data and infer effects from observed correlations. This approach makes conclusions vulnerable to selection bias. The second problem is rooted in the difficulty to empirically separate the effects of someone's education, occupation, and of mismatches proper, since they are linearly dependent: a mismatch is the difference between education and occupation (Blalock 1966). To identify effects, previous work had to rely on strong assumptions about the nature of mismatch-effects. These assumptions, however, were not explicitly justified but hidden in the mechanics of the respective statistical model used. The result of these $a d-h o c$ fixes has been a sharp disagreement in main conclusions between different studies.

This study reviews earlier efforts to model mismatch effects and makes their sometimes questionable theoretical positions explicit. In a second step, I propose a novel methodology to addresses the problems of previous research. Firstly, I provide the only analysis of the effect of mismatches on social and political attitudes exploiting longitudinal data. Secondly, I tackle the fundamental identification problem in mismatch research by building on recent advances in the modelling of age, period and cohort effects, which suffers from a similar identification problem (Fosse and Winship 2019b). My approach makes explicit use of relatively weak, and more importantly, 
transparent assumptions about the data generating process to partially identify mismatch effects. Throughout, I focus on social and political outcomes, which, once at the center of debate, have received relatively little attention in recent research.

I employ data from two comparable longitudinal population surveys with large sample sizes, the United Kingdom Longitudinal Household Study (UKLHS; Buck and McFall 2011) and the German Socio-Economic Panel Study (GSOEP; Deutsches Institut Für Wirtschaftsforschung 2017). The UK and Germany are interesting cases to study, because of the large differences that exist in their organization of labor markets, education systems and political cleavages. Comparing results across these two very different countries can serve as a first test into the generalizability of my main findings. If there is any contextual variation in the relationship between mismatches and political attitudes, I would expect it to be present in this comparison. Vice versa, if the results prove to be similar in these countries, they should generalize to other (Western) countries, too.

Overall, the results of my analyses document that qualification-job mismatches are highly consequential for the economic and subjective well-being of individuals, even net of the main effects of education and occupation. However, the analyses also provide evidence that the consequences of mismatches for the political domain have been overstated in previous research.

\section{Theory and previous research}

In this section, I first discuss the original hypotheses put forward by status inconsistency theory (SIT) and their empirical record, before I explain the fundamental identification problem using a numerical example. In a next step, I then examine previous approaches to handle the problem for their theoretical content. Finally, I propose a bounding approach to estimating mismatch effects, which avoids some of the pitfalls of older work.

\section{Status inconsistency theory}

Why should a qualification mismatch result in stress, dissatisfaction, social withdrawal, and political alienation? Qualification mismatches were first investigated as a source of political dissatisfaction in the context of sociological status inconsistency theory (Lenski 1954). SIT originated in the post-war United States and had important conceptual affinities to role theory, Parsonian functionalism, and the social psychology of the time (see Stryker and Macke 1978 for a review). 
The micro-mechanism suggested by SIT starts from the premise that actors seek to achieve cognitive consonance in their self-image, and that this is hampered by incongruous positions on different dimensions of social status. According to SIT, four channels through which status inconsistency ${ }^{2}$ creates psychological stress and eventually results in political unrest can be distinguished: First, status inconsistency creates cognitive dissonance through uncertainty about one's identity, which leads to stress (Festinger 1962; Geschwender 1967; Jackson 1962). Second, status inconsistency makes it hard for others to determine the appropriate role of actors in social interactions, and hence make it less likely that actors experiences interactions as rewarding (Lenski 1956). Third, status inconsistency in terms of education and occupation can take the form of overqualification, which implies that past expectations about the future, as instilled by education and training, have not been realized. Such "underrewarded inconsistency" leads to frustration (Geschwender 1968). The fourth and final causal relationship hypothesized by SIT is that status inconsistent individuals will eventually externalize these sources of stress and seek to change the social environment that they blame for their dissatisfaction (Goffman 1957). Originally, analysts hypothesized that this would result in left-wing activism and voting, but other contributions also argue that frustration can be expressed by endorsing far-right politics (Portes 1972; Stryker and Macke 1978).

It is worth noting that each of the four causal channels in original SIT has implications that are to a large degree testable in separation. The first channel implies that any mismatch should lead to dissatisfaction and stress, regardless whether it is one of over- or underqualification. It is the absolute difference between actual and common education that matters, regardless of its sign. The second channel implies that mismatch should affect not only cognitive states, such as satisfaction, but have effects on social behavior, e.g. membership in voluntary organizations, as well. The third channel implies that effects of overqualification should be stronger than those of underqualification, since it is especially when realized states fall short of anticipated ones that disappointment can be expected. The combination with the first channel thus suggests a pattern where both types of mismatch affect satisfaction negatively, but more strongly for overeducation. The fourth channel, finally, is in operation, when the effects of mismatches exceed the personal level and affect political and social attitudes and behaviors.

What empirical evidence on these dynamics has mismatch scholarship produced? The SIT literature has resulted in a large number of inconsistent findings,

${ }^{2}$ I will use the terms inconsistency and mismatch interchangeably. I speak of inconsistencies, when I refer to the SIT-literature, and of mismatches, when other scholarship is concerned. 
with some reporting strong evidence for (Geschwender 1968; Goffman 1957; Jackson 1962; Jackson and Burke 1965; Lenski 1954, 1956; Vaisey 2006; Zhang 2008), and others reporting strong evidence against mismatches as sources of personal and political discontent (Blocker and Riedesel 1978; Jackson and Curtis 1972; Olsen and Tully 1972; Portes 1972).

In the following I will explain, why this lack of agreement is rooted in conceptual and methodological difficulties in defining and modelling mismatches (Blalock 1966; Duncan 2005; Hope 1975; Lenski 1964; and Sobel 1981). As I will elaborate, these complications also put the evidential value of the existing empirical literature into question.

\section{The fundamental identification problem of mismatch theory}

Conceptual and methodological difficulties in inconsistency research are due to a fundamental identification problem. This issue is often regarded as a merely methodological one, but my intention is to show that it cannot be separated from theory. To clarify this claim, I distinguish between three levels: the actual data generating process (DGP) in reality, the theoretical "structural model" of that process, and the empirical ("reduced form") model which is statistically estimable.

The basic problem is already apparent in Lenski's seminal statement of the basic hypothesis of status inconsistency research: "individuals characterized by a low degree of status [consistency] differ significantly in their political attitudes and behavior from individuals characterized by a high degree of status [consistency], when status differences in the vertical dimensions are controlled." (Lenski 1954:405f., my italics). The key point in this statement is that a third variable - the degree of consistency, which is itself a function exclusively of education and occupation - is proposed to influence experiences, attitudes, and behavior, net of education and occupation.

Applied to an example, Lenski's hypothesis suggests that a lower-level hotelmanager with a college degree in business administration experiences more stress than one, who underwent the vocational training typical for his position, conditional on their respective actual levels of education, and that this is because the former perceives a stressful difference between his high-status education and his relatively lower status occupation, whereas the two fall together for the latter.

In order to illuminate the fundamental identification problem, I now introduce a simple formal framework to represent Lenski's conjecture. I concentrate on two dimensions of status, education and occupation, and on linear relationships. This is 
because the identification problem is limited to the linear components of the relationships. Any non-linear deviations from them are identified without problems, a fact I discuss below (see also Fosse and Winship 2019b).

A linear version of Lenski's hypothesis can be represented as proposing a nonzero $\beta_{M M}$ in the model

$$
Y=\beta_{E} X_{E}+\beta_{O} X_{O}+\beta_{M M}\left(X_{E}-X_{O}\right)
$$

where $Y$ is the outcome of interest, and $X_{E}$ and $X_{O}$ are education and occupation, two different metric z-standardized dimensions of social status, for instance job prestige and years of education. Since they are standardized, they indicate an individual's relative position on that dimension in the population. $\left(X_{E}-X_{O}\right)$ represents the linear mismatch term. It is positive for over- and negative for underqualified workers. Eq.1 is best thought of as a structural model of the true DGP: $Y$ is produced from combinations of $X_{E}$ and $X_{O}$ according to the parameters $\beta_{E}, \beta_{O}$, and $\beta_{M M}$.

Returning to my example, the term $\left(X_{E}-X_{O}\right)$ is zero for the hotel manager with the required vocational education, because the relative status of his education and his occupation are identical. However, $\left(X_{E}-X_{O}\right)$ is positive for the college graduate, because the relative status of his occupation is lower than that of his education. If $\beta_{M M}$ is nonzero, as hypothesized by Lenski, this third term will affect $Y$ above and beyond $X_{E}$ and $X_{O}$ for the mismatched graduate.

The framework of Eq.1 is important, because it shows that the structural model proposed by Lenski is empirically unidentified. Three distinct parameters $\left(\beta_{E}, \beta_{O}, \beta_{M M}\right)$ govern the relationship between just two independent variables ( $X_{E}$ and $\left.X_{O}\right)$ and the outcome (Blalock 1966). This means that given identical combinations of education and occupation $\left(X_{E}\right.$ and $\left.X_{O}\right)$, an infinite number of combinations of the structural parameters $\beta_{E}, \beta_{O}$, and $\beta_{M M}$ could theoretically result in the same $Y$. For the hotel-managers, this means that the same observed values of stress (e.g. $Y_{\text {matched }}=$ $10 ; Y_{\text {mismatched }}=11.25$ ) could result from identical independent variables (e.g. with the relative statuses as $X_{E, \text { college }}=15 ; X_{E \text {,voctrain }}=10$ and $X_{O \text {, hotel man. }}=10$ ) through radically different data generating processes (e.g. with $\beta_{E}=0.25, \beta_{O}=0.75$, and $\beta_{M M}=0$ or with $\beta_{E}=-0.25, \beta_{O}=1.25$, and $\left.\beta_{M M}=0.5\right){ }^{3}$

\footnotetext{
${ }^{3}$ This is shown by the following simple calculations, which plug in the respective values into Eq. 1., once for the first set of structural parameters $(2 \mathrm{a}$ and $2 \mathrm{c})$ and once for the second set $(2 \mathrm{~b}$ and $2 \mathrm{~d})$ :$$
Y_{\text {matched }}=10=.75 * 10+.25 * 10+0(10-10)
$$$$
=1.25 * 10-0.25 * 10+0.5(10-10)
$$$$
Y_{\text {mismatched }}=11.25=.75 * 10+.25 * 15+0(15-10)
$$$$
=1.25 * 10-0.25 * 15+0.5(15-10)
$$ 
For a researcher who observes $X_{E}, X_{O}$ and $Y$ and wants to understand the true DGP as represented by the structural model in Eq. 3-1, it is therefore never possible to decide without further assumptions, whether the relative stress levels of the collegeeducated manager compared to the vocationally trained one are affected by his mismatch, or exclusively due to processes resulting in higher levels of stress among the higher educated.

At its core, this fact is due to the nature of the DGP, but its implications appear as methodological problems to researchers. The fundamental problem of mismatch research is that because different structural parameters can produce the same data, the data and empirical models alone cannot reveal the true DGP. Data alone can therefore never provide an answer as to whether mismatches indeed have effects on social and political outcomes. Thus, in order to identify mismatch effects, assumptions about the DGP, that is restrictions on the structural model of one kind or another, are indispensable. As I will show below, such theoretical assumptions are present even in the approaches which try to hide them. However, good scientific practice asks to justify constraints on substantive grounds and to make them transparent. The identification problem that mismatch research has faced cannot therefore be solved by methods, it must be solved by leveraging prior knowledge with careful and transparent theoretical examination

\section{Theories about the structure of mismatch effects}

Throughout the last 60 years, different fields with different research questions have approached mismatch-effects. The different strategies to study mismatch effects were often framed as purely methodological proposals. But really they are theoretical statements about the process that generates the data. Theoretical models like Eq.1 do not have in themselves a unique solution in terms of $\beta_{E}, \beta_{O}$, and $\beta_{M M}$. Previous strategies achieved a unique solution only by implicitly assuming various structures that differ from that in Eq. 3-1. The fact that different proposals lead to different estimates of $\beta_{E}, \beta_{O}$, and $\beta_{M M}$ resulted in the confusion that has plagued the literatures on mismatches since the early 1960s. Even worse, as I have shown above, these estimates cannot be distinguished on empirical grounds, because they are compatible with the same data. What is needed, therefore, is a review of the theoretical commitments different research strategies imply and an informed discussion in which situations they are appropriate. In the following, I offer such a discussion. 


\section{Sociologists and their cross-tables}

An important class of early strategies to test for mismatch effects were the so-called square additive models. Originally, they were formulated in a cross-table context, but here I translate them into a linear equation framework, with which modern researchers are more familiar. These empirical models compare the variance explained by a baseline model of additive main effects of education and occupation $\left(\beta_{E}\right.$ and $\beta_{O}$ in above model),

$$
Y=\beta_{E} X_{E}+\beta_{O} X_{O}+\epsilon
$$

with the variance explained by models that allow for separate inconsistency parameters (e.g. dummies for over- and underqualified workers) (Duncan 2005:90ff.). After fitting a baseline model like Eq. 3-2, researchers regarded significant effects of such dummy-variables as evidence of inconsistency effects.

But recalling the example for the case of two hotel managers, data which was in fact generated by linear mismatch effects can easily be described using a constrained baseline model like Eq. 3-2 (compare footnote 3). However, this comes at the price of estimates of the remaining $\beta \mathrm{s}$ that do not correspond to the true DGP. The estimates of the main effects in the baseline model will absorb the linear component of mismatch effects. This is problematic, because what is left for the additional mismatchparameters to pick up in additive models are merely the non-linear components of mismatch-effects. All linear components are contained in the baseline estimates. 


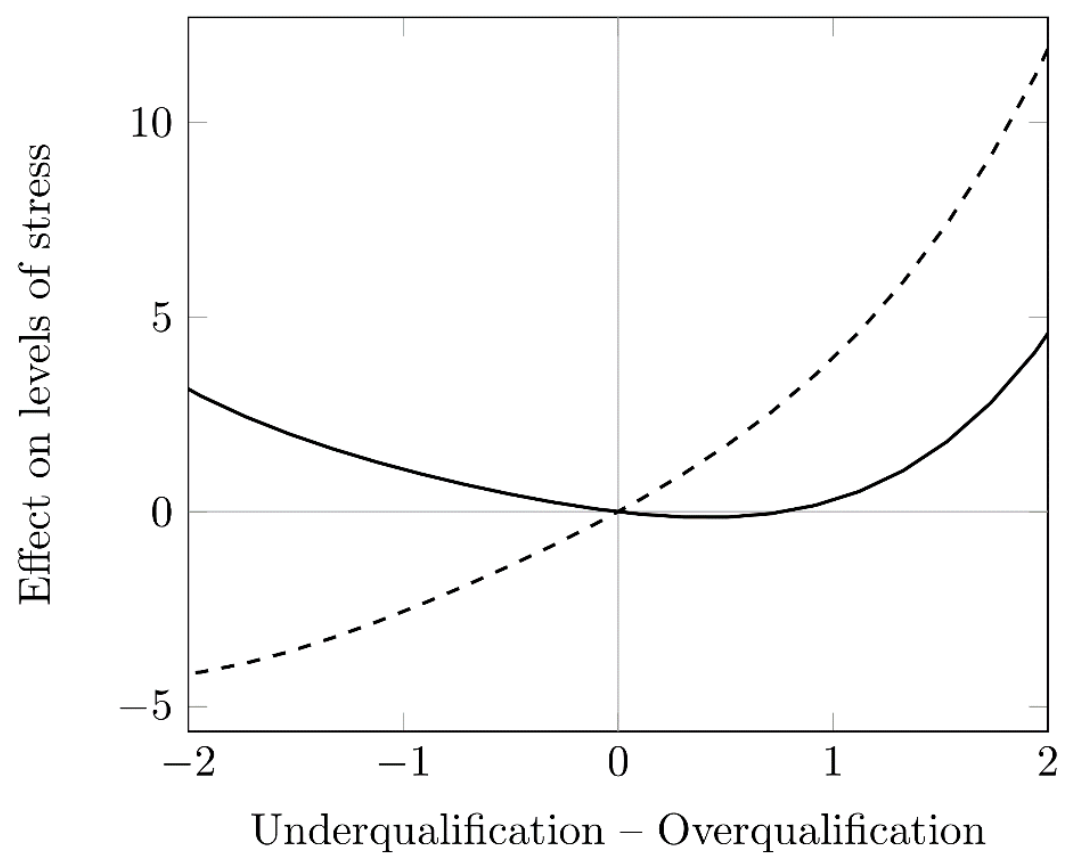

Figure 0-1: Illustration of the bias introduced by square additive models

Omitting the linear component may give a very distorted picture of the true effects of mismatches. This is illustrated by the fictitious example in Figure 3-1. Assume the values of stress on the $y$-axis are in reality produced from the values of mismatch on the $\mathrm{x}$-axis according to the DGP represented by the dashed line. We see decreased stress among the under- and increased stress among the overeducated. The mismatch effects an additive model will return, on the other hand, are given by the solid line. These estimates, or any approximation of them, only contain the non-linear components of the true mismatch effects. Based on these values, we would wrongly conclude that underqualification has a stress-increasing effect, whereas the true stressenhancing effect of overqualification would be underappreciated. ${ }^{4}$

Square additive models are an example of a strategy to identify point estimates of mismatch effects that rely on a theoretical commitment to one of the structural linear components in Eq.1 being zero in reality. If this assumption is true, one of the three terms of Eq. 3-1 vanishes, and the two remaining structural parameters match two independent variables and can be uniquely and unbiasedly estimated from the

\footnotetext{
${ }^{4}$ In this example, the true relationship between mismatch and stress, net of education and occupation, is given by $Y=0.09\left(X_{E}-X_{O}\right)^{4}+0.25\left(X_{E}-X_{O}\right)^{3}+0.6\left(X_{E}-X_{O}\right)^{2}+3\left(X_{E}-X_{O}\right)$, whereas the square additive model would return at best $Y=0.09\left(X_{E}-X_{O}\right)^{4}+0.25\left(X_{E}-X_{O}\right)^{3}+$ $0.6\left(X_{E}-X_{O}\right)^{2}-0.65\left(X_{E}-X_{O}\right)$. This is because the linear approximation of the true relationship is $Y=3.65\left(X_{E}-X_{O}\right)$ and will be absorbed by the vertical dimensions.
} 
data. If it is not true, however, the structural model that is being parametrized is different from the process that produced the data, resulting in the problems I have illustrated.

If the goal is to learn about the true DGP, strong assumptions like those embedded in additive models should be carefully defended. In general, there is arguably no good reason to believe that there is no linear mismatch parameter in the structural model. After all, the existence of these effects is what the empirical examination is supposed to reveal. As has been noted before, this makes the results of the square additive model literature questionable (Blalock 1966; Hendrickx et al. 1993; Hope 1975; Sobel 1981).

As a reaction to these problems, the so-called diamond model, which allows linear mismatch effects in the structural model, was proposed (Hope 1975). However, to achieve this, Hope had to reformulate the structure implied by Eq. 1 in a way that amounts to a full-blown restatement of SIT. In Hope's model, any multidimensionality of social status that is consequential for an outcome appears as an inconsistency effect.

Hope regards social status as a latent, vertical, unidimensional construct. In practice, this general status index is constructed as some weighted average of, for instance, education and occupation. According to Hope, inconsistency refers to any non-zero value on a dimension of social status apart from this general vertical index. Such non-vertical dimensions could be, for example, the variances in education or occupation that do not fully align with general status. In other words: As soon as the constituent empirical referents of the general status dimension (say a diploma or a job role) do not correlate perfectly, agents are thought to feel strain from inconsistent statuses. But such a view leaves open, how the latent status dimension proposed by Hope is supposed to become socially effective or even be perceived by actors. So while parameters of the structural model implied by the diamond model may be estimated from data without problems, it comes at the cost of positing an unobservable, intangible concept and an unspecified causal mechanism.

Technically, the separation into vertical and non-vertical components can be achieved by the cross-table techniques (the diamond-model) described in Hope (1975) or by principal component analyses and similar methods. However, it is important to note that such procedures do not offer any new identifying information. They merely rotate the existing status matrix and relabel the dimensions. In Hope's example of a diamond model, the columns of the rotated matrix, (i.e. the first variable in a linear model) represent the vertical status, defined simply as the mean of the two main dimensions, and the rows (i.e. the second variable) indicate linear inconsistency 
values, defined as the difference between the constituent dimensions, e.g. education and occupation.

For the case of hotel managers, this would mean that the matched manager receives a vertical status score of $10((10+10) / 2)$ and an inconsistency values of 0 $((10-10) / 2)$, the mismatched manager a vertical status of $12.5((10+15) / 2)$, and an inconsistency of $2.5((15-10) / 2)$. In this example, Hope's model would return an inconsistency parameter of $-0.5 .^{5}$ Note that in this model, the higher level of stress in the mismatched manager will be regarded as evidence of inconsistency effects, regardless of whether it is due to a separate mismatch-parameter in the sense of Eq. 3-1 or simply due to an independent effect of education, net of occupation (compare footnote 3). More generally, Hope's model will return inconsistency effects, whenever the main dimensions of Eq. 1 differ in the strength of their association to the outcome. This criterion for inconsistency effects is clearly weaker and substantively different from that implied by Lenski's original formulation.

Economists and the refined Mincer equation

A second perspective on mismatch-effects emerged in the 1980s among economists, who saw them as a way to test different theories of labor market allocation. The socalled ORU decomposition (Over-, Required, and Undereducation) splits up the attained education $(\mathrm{E})$ term in a wage equation into three components: required education, the amount of education that is required in a worker's job $(R$, to which I here refer to as $O$ for "occupation" in order to maintain consistency with the SIT literature); overqualification $(O Q)$, the years of education of a worker beyond of what is required, and underqualification $(U Q)$, the years of education a worker is short of requirements, such that $E=O+O Q-U Q$, where $O Q=E-O$, if $E-O>$ 0 and 0 otherwise, and

$U Q=O-E$, if $O-E>0$ and 0 otherwise, resulting in the wage equation

$$
Y=\beta_{0}+\beta_{O Q} O Q+\beta_{O} O+\beta_{U Q} U Q+\epsilon
$$

(Duncan and Hoffman 1981).

In an important advance over the classic Mincer equation, the $O Q$ and $U Q$ parameters in this specification allow analysts to investigate whether mismatched workers differ from matched workers with respect to $Y$ in an occupation with the same education requirements.

\footnotetext{
${ }^{5}$ The respective equations are $11.25=1 * 12.5-.5 * 2.5$, for the mismatched worker, and $10=1 *$ $10-.5 * 0$, for the matched worker.
} 
From the perspective of the general framework of Eq. 3-1, Eq. 3-3 amounts to omitting $\beta_{E} X_{E}$, the term that captures the main effect of education. The ORU decomposition is hence another example of an identification strategy that proposes one linear term of Eq. 1 to be zero in the structural model. In addition, Eq. 3 splits up the term $\beta_{M M}\left(X_{E}-X_{O}\right)$ into $\beta_{O Q} O Q$ and $\beta_{U Q} U Q$, that is, it allows for a nonlinearity in mismatch effects. Since only two linear effects, $\beta_{O}$ and the shared linear component in $\beta_{O Q}$ and $\beta_{U Q}$ have to be estimated, the model is uniquely identified.

The structural model implied by this strategy is one, where past education does not play a role beyond occupational positions and potential mismatch. Applied to the hotel manager example, all differences in stress between the matched and the mismatched manager are regarded as stemming from their differing mismatch status - but not from their differing education. In this view, an enduring, independent role of socialization through education for later life outcomes is excluded.

How convincing is such a perspective on labor market careers? Arguably, this very much depends on the outcome of interest. We have to distinguish between outcomes that result exclusively from the current employment situation and outcomes that reflect a more complex layering of experiences over the life-course. For instance, decades of research have documented that many political and social attitudes and behaviors are relatively stable and partially formed early in life, among other things by educational experiences (for reviews, see Sears and Brown 2013). For these outcomes, a structural model as proposed by ORU models seems to poorly reflect reality. However, for outcomes, which economists have investigated with it, a structural model in the form of an ORU model is much more plausible. It is difficult to conceive of a causal influence on an employee's wages that is not fully mediated through properties of her current employment situation. So, while ORU models are plausible in typical applications in economics, they should not unthinkingly be applied in sociological research.

\section{Recent trends}

The most recent studies of mismatch effects have abandoned the traditional cross-table models of mismatch effects and instead relied on more flexible regression techniques. Public health researchers, for instance, have rediscovered the original claims of SIT and produced a series of studies that link qualification mismatch to higher levels of stress, poorer self-rated health, and increased mortality (Dudal and Bracke 2019; Dunlavy, Garcy, and Rostila 2016; Smith et al. 2012; Zhu and Chen 2016). Some of these studies claim that mismatches have potentially important health-consequences, which may be associated with decreased social and political activity. Studies in this 
literature are of course equally affected by the identification problems I have pointed out. Closer examination reveals that the empirical models in this literature assume different structural models, most often ones, which exclude the main effect of occupation. They thereby assume that people's current occupation is unrelated to their health status - an unlikely situation. It is hence not clear, how much evidence of the health consequences of qualification-mismatches, net of occupation, there actually is.

In sociology, two papers have renewed the discipline's longstanding interest in inconsistency effects. In the first, Vaisey (2006) claims that workers in the United States, who are overqualified are more politically liberal and less achievement oriented than workers in a similar job, who are not inconsistent. His work relies on the ORU decomposition. This approach is descriptively valid and informative. But as I have argued above, from a DGP perspective it is questionable, whether the structural model implicit in ORU approaches (i.e. one that assumes that effects of education are fully mediated by occupation) is a valid representation of the process of attitude formation. In the second, Zhang (2008) explicitly proposes a test of SIT for the case of inconsistencies in income and education. However, his empirical models are only identified, because he constrains the effect of inconsistency to have the same sign, regardless of the direction of a mismatch. While a symmetric mismatch-term (strictly equal effects of under- and overqualification, $\beta_{O Q}=\beta_{U Q}$ ) is a feature of Lenski's original statement of SIT (he assumed a squared relationship between outcomes and mismatch, which implies symmetry), I think that its existence should be concluded $a$ posteriori from the data, rather than required a priori for a method to work. Note that similar to the square additive model, the assumption of symmetric effects of inconsistency claims that the linear component of the mismatch effect is zero in the DGP. Hence, all the caveats I discussed above apply. It is unclear in how far the results in Zhang (2008) depend on these problematic assumptions.

\section{A bounding approach to mismatch-effects}

My literature review has shown that past attempts to test for mismatch effects were implicitly wed to very specific theoretical models of the mismatch process. In the following I introduce a new approach to investigate mismatch effects that allows us to use prior knowledge and theoretical analysis to flexibly and transparently specify a theoretical model that better reflects the true DGP.

To do this, I follow recent work of Fosse and Winship in the context of modelling age, period, and cohort (APC) effects (Fosse and Winship 2019a, 2019b). The bounding-approach developed by Fosse and Winship (2019b) is based on the idea that 
prior knowledge can be used to formulate explicit constraints on the parameters of a structural model that is empirically non-identified. If some values of structural parameters can be discarded a-priori on theoretical grounds, this limits the range of values other parameters in the model can take.

The starting point of a bounding analysis is that even though single parameters of a general structural APC- or a mismatch-model are not uniquely identifiable from the data, combinations of them are (O'Brien 2014). In the case of mismatches, we can identify the empirical parameters $\theta_{1}$ and $\theta_{2}$, with

$$
\begin{gathered}
\theta_{1}=\beta_{E}+\beta_{O} \text { and } \\
\theta_{2}=\beta_{O}-\beta_{M M},
\end{gathered}
$$

where the $\beta$ s are the structural coefficients from Eq. 3-1 (Fosse and Winship $2019 \mathrm{~b}$ ). The fact that $\theta_{1}$ and $\theta_{2}$ are uniquely identified creates dependencies in the parameter space that analysts can exploit to arrive at partial identification of a structural parameter of interest. By making an informed assumption about the sign and potentially the magnitude of $\beta_{E}$ and $\beta_{O}$, it is possible to create finite bounds around empirical estimates of $\beta_{M M}$. By rearranging Eq. 3-5 and substituting $\beta_{O}$ in Eq. 3-4 we get two restrictions which $\beta_{M M}$ has to satisfy:

$$
\begin{gathered}
\beta_{M M}=\beta_{O}-\theta_{2} \text { and } \\
\beta_{M M}=\theta_{1}-\theta_{2}-\beta_{E} .
\end{gathered}
$$

If it can now be assumed that $\beta_{O}$ is larger than some minimal value, $\beta_{O}>\beta_{O}^{\text {min }}$, and similarly that $\beta_{E}>\beta_{E}^{\text {min }}$, we know that

$$
\beta_{O}^{\text {min }}-\theta_{1}<\beta_{M M}<\theta_{1}-\theta_{2}-\beta_{E}^{\text {min }},
$$

which represent the bounds within which the true linear mismatch effect lies. In other words: if prior knowledge and theoretical examination suggest that the true main effects of education and occupation are larger than some values, this results in finite bounds for the structural mismatch effect. The same holds if both education and occupation effects are negative and can be assumed to be below some value. Instead of relying on implicit and ad-hoc constraints to arrive at point identification, the Fosse and Winship approach allows using weaker, theoretically justifiable, and, most importantly, transparent constraints to identify a range of values for the parameters of 
interest, which are consistent with the theoretical assumptions (represented by $\beta_{O}^{\min }$ and $\beta_{E}^{\min }$ ) and the data (represented by $\theta_{1}$ and $\theta_{2}$ in Eq. 3-8).

What do we know about the relative importance of education and occupation?

Since they define the structural model, the identifying assumptions in Eq. 3-8, $\beta_{O}^{\min }$ and $\beta_{E}^{\text {min }}$, have to be carefully specified. Large literatures in sociology and political sciences have shown that social and political attitudes and behaviors vary strongly and partially independently with education and occupation (e.g. Niemi and Sobieszek 1977; Verba, Nie, and Kim 1978). Extant research is also clear about the fact that education and occupational status co-vary with our outcomes of interest in the same directions. Therefore Eq. 3-8, which requires that both main effects have the same sign, is applicable: the theoretical constraints I impose on the structural model in Eq. 3-1 will result in finite bounds for the estimates of the linear mismatch parameter $\beta_{M M}$ in Eq. 3-1 for all of our variables of interest.

But recalling my discussion of the applicability of ORU models in different domains, we can go one step further. On the basis of substantive reasoning, it is possible to distinguish between two types of dependent variables, those with a socialization component, where experiences made during the education-phase are likely to have a lasting impact, and those that are produced directly by actors' experiences and behavior in the workplace. As I have argued, it is theoretically hard to conceive of a scenario in which education directly (that is, net of occupation and mismatch) affects wages and, arguably, job satisfaction. For this second type of outcome, there is more specific prior knowledge than for the first type, where we usually just know that both education and occupation have some non-trivial effect of the same direction. As a result, I am able to present plausibly point-identified estimators of mismatch effects on outcomes of the second type.

But what about the first type of variables? Here, the width of the bounds, that is the amount of information conveyed, partially depends on the amount of prior information available. However, it is difficult to extract from existing research precise lower bounds for main effects of education and occupation. In the main text, I employ the following relatively conservative constraints:

$$
\frac{\beta_{E}}{3}<\beta_{O}^{\min }<3 \beta_{E} \text {, which implies that } \frac{\beta_{O}}{3}<\beta_{E}^{\min }<3 \beta_{O}
$$

In other words: I assume that the effect of occupation is at most three times as strong as that of education and vice versa. This leaves plenty of room for empirical differences to play out: If $\beta_{O}$ were $1, \beta_{E}$ could range between 0.3 and 3 . While 
plausible, this choice of relative weights is admittedly arbitrary. I therefore present results obtained using other, even less restrictive values in Supplement D.

To sum up, in contrast to earlier efforts, which assumed an arbitrarily stunted versions of Eq. 3-1, I propose to work with a structural model that preserves all the features of the general model in Eq. 3-1. Instead of indiscriminately claiming that one entire term is zero, as most previous approaches implicitly did, I merely claim that both occupation and education have some association with our outcomes - except in cases, where substantive reflection suggests otherwise.

\section{Data and methods}

I base my empirical estimates of mismatch effects on two harmonized data sources from two countries: the UK Longitudinal Household Study 2009-2016 (UKLHS; Buck and McFall 2011) and the German Socio-Economic Panel Study 1984-2016 (SOEP; Deutsches Institut Für Wirtschaftsforschung 2017). UKLHS and SOEP are comparable sources of data in that both are longitudinal surveys of private households with high-quality fieldwork. Both studies rely on similar sampling strategies, questionnaire design, and often even use the same items.

I restrict the analytical sample to non-self-employed working men and women between the ages of 20 and 60, who are not currently enrolled in full-time education or training. For the cross-sectional analysis, I restrict the SOEP sample to the years after 2004, because important control variables were collected only after that date. Throughout, I employ the post-stratification weights provided with the data to account for unequal sampling and attrition probabilities.

\section{Measuring education, occupation and mismatch}

I rely on a generalized version of the ORU decomposition to model the effects of vertical qualification mismatch that allows including a separate term for the main effect of education. My central independent variables are therefore measures of actual education $(E)$, required education in someone's occupation $(O)$ and of mismatch. In line with the ORU tradition, I rely on virtual years of education and the so-called realized matches approach to identify the required education in an occupation (see McGuinness 2006 for an overview of measurement approaches, and Section A in the Online Supplement for details on the coding of years of education). Concretely, I distinguish occupations using the 3-digit ISCO88 classification and estimate the mean years of education in each occupation in the post-stratified UKLHS and SOEP samples 
as a measure of required education. Overqualification $(O Q)$ and underqualification $(U Q)$ are defined as explained above. Note that while $O$ is regarded in the ORU tradition as a measure of qualification requirements, it can just as well be interpreted as a measure of occupational status as in the SIT-tradition. Indeed, the empirical correlation between the occupation mean years of education and the ISEI, an accepted measure of occupational status, is $r=0.87$ in Germany and $r=0.81$ in the UK in the respective 2014 waves of my sample.

\section{Control variables}

In the cross-sectional analysis I control for a rich set of personal characteristics, including age, age-squared, measures of cognitive ability, BIG-5 personality, risk aversion, locus of control, parental occupation, parental education, immigration background, gender, as well as for region and survey year. Details on the measurement of these variables can be found in Online Supplement A. I also ran separate analyses by gender, but results were largely identical. Gender specific results can be found in Supplement C. In the longitudinal models, I only adjust for age, the tenure in the current position, and survey year.

Not all controls were measured in all years or for all respondents. If information is missing, I carry forward the latest observation of a respondent. If a control variable has never been measured, I rely on 10 imputations from a chained equations model (Van Buuren 2012). The multiple imputation models take into account all variables that feature in the analysis models and additional variables that may help to reduce prediction uncertainty.

\section{Outcomes}

In order to comprehensively capture the relevance of mismatch, I consider nine different facets of social, political and occupational behaviour, involvement, identities and satisfaction, as well as trust and income. While the items I rely on are designed to capture identical concepts in the two surveys, it is important to note that sometimes the wording is not strictly identical in SOEP and UKHLS. The Online Supplement A documents the questions and response categories used in the two countries.

Table 3-1 shows how many data points, from how many respondents the two datasets provide on these variables. These figures equal the sample sizes my models can draw on. Since many variables were collected in different waves, my analytical samples differ for different dependent variables, and in Germany for different specifications. 
Trust, satisfaction with democracy, job and life satisfaction, and the respective importance of politics/one's profession were measured using standard Likert scales in both questionnaires. In order to increase comparability between these measures, I zstandardize them, so that one unit corresponds to one sample specific standard deviation.

Left vote, far-right vote, and member of an organization are binary variables that indicate whether a respondent expresses left-wing/far-right voting intentions or reports being the member of at least one organization. I refer readers to Supplement A for details on my coding of parties.

My last dependent variable is hourly gross labour income. I derive this variable from the imputed gross labour incomes in the datasets, which I divide by the reported contracted monthly working hours. I report results for the unstandardized natural logarithm of this variable. 


\begin{tabular}{|c|c|c|c|c|c|c|c|c|c|c|}
\hline & Trust & $\begin{array}{c}\text { Satisfaction } \\
\text { democracy }\end{array}$ & $\begin{array}{c}\text { Job } \\
\text { satisfaction }\end{array}$ & $\begin{array}{c}\text { Life } \\
\text { satisfaction }\end{array}$ & $\begin{array}{c}\text { Importance } \\
\text { politics }\end{array}$ & $\begin{array}{l}\text { Importance } \\
\text { profession }\end{array}$ & $\begin{array}{l}\text { Logged hourly } \\
\text { wages }\end{array}$ & $\begin{array}{c}\text { Vote left } \\
\text { party }\end{array}$ & $\begin{array}{l}\text { Vote far-right } \\
\text { party }\end{array}$ & $\begin{array}{c}\text { Member } \\
\text { organization }\end{array}$ \\
\hline & 14 & & & & & & & & & \\
\hline Nobs & $\begin{array}{r}789 \\
14\end{array}$ & 22112 & 84428 & 77446 & 20528 & 21599 & 84661 & 59687 & 59687 & 11838 \\
\hline $\mathrm{N}_{\text {Resp }}$ & 789 & 14964 & 25955 & 24334 & 14631 & 15227 & 25990 & 20749 & 20749 & 11838 \\
\hline $\begin{array}{l}\text { Mean number of } \\
\text { waves/person }\end{array}$ & 1 & 1.47 & 3.25 & 3.18 & 1.40 & 1.41 & 3.25 & 2.87 & 2.87 & 1 \\
\hline Longest gap & - & 2 & 0 & 0 & 2 & 2 & 0 & 0 & 0 & - \\
\hline & 25 & & & & & & & & & \\
\hline Nobs,pooled & $\begin{array}{r}771 \\
17\end{array}$ & 17353 & 124858 & 124858 & 40624 & 40682 & 122666 & 45928 & 45928 & 21368 \\
\hline $\mathrm{N}_{\text {Resp,pooled }}$ & $\begin{array}{r}383 \\
14\end{array}$ & 13331 & 28377 & 28377 & 22597 & 22619 & 27769 & 14157 & 14157 & 14332 \\
\hline Nobs,FE & $\begin{array}{r}977 \\
11\end{array}$ & 22112 & 202729 & 205849 & 45387 & 45387 & 201381 & 83225 & 83225 & 34436 \\
\hline$N_{\text {Resp,FE }}$ & 825 & 14964 & 36625 & 37491 & 23358 & 23358 & 36730 & 20707 & 20707 & 18040 \\
\hline $\begin{array}{l}\text { Mean number of } \\
\text { waves/person }\end{array}$ & 1.26 & 1.47 & 5.53 & 5.49 & 1.94 & 1.94 & 5.48 & 4.01 & 4.01 & 1.90 \\
\hline Longest gap & 4 & 4 & 0 & 0 & 4 & 4 & 0 & 0 & 0 & 4 \\
\hline
\end{tabular}

Table 0-1: Sizes and characteristics of analytical samples 


\section{Modelling strategies}

I proceed in two steps. In the first, I estimate a generalized version of the crosssectional ORU decomposition on pooled samples. In the second, I estimate a specification of this model that includes person-specific fixed-effects and hence rules out confounding by unobserved time-constant variables.

The model I estimate in the first step is given by the equation

$$
Y=\beta_{0}+\beta_{O} O+\beta_{E} E+\beta_{O Q} O Q+\beta_{U Q} U Q+\sum \beta_{X_{j}} X_{j}+\epsilon,
$$

where $O, E, O Q, U Q$ refer to the parameters discussed above and $X_{j}$ is the $\mathrm{j}^{\text {th }}$ control variable. This specification corresponds to a general structural model, which contains a perfect linear dependency between $O, E$, and the shared linear component of $O Q / U Q$. Eq. 10 is partially identified through the constraints in Eq. 3-9. I refer to this as the E-ORU specification, since it contains all four terms of $E, O Q, O$ and $U Q$ simultaneously. Note that I report results with $O, E, O Q$, and $U Q$ in a years-ofeducation-metric, while the constraints in Eq. 3-9 refer to standardized coefficients.

In the E-ORU specification, the bounded OQ and UQ parameters reflect the change in the outcome associated with one additional year of under- or overeducation, net of actual education, required education, and other covariates. The E-ORU model is estimated using constrained least squares. I base inference on standard errors that are clustered at the person-level.

The pooled-data E-ORU specification addresses the linear dependency of O, E and MM. However, it is still susceptible to selection bias. I address this problem using a person-fixed-effects (FE) approach. This design eliminates all time-constant confounders that might bias the relationship between mismatches and outcomes.

In this step, I make use of all the survey years available to us, in which the respective dependent variable was measured, i.e. I use all waves of the GSOEP since 1984. Table 3-1 lists the number of gap years between measurements for the dependent variables, and the mean number of observations per respondent that I draw on to estimate the FE models. I only make use of data from respondents, whose education has remained constant throughout the observation period and use only 
mismatch-changes that I can relate to job-changes as indicated by changes in the 3digit ISCO occupational title, ignoring periods of unemployment and inactivity.

If the E-ORU specification is applied in a FE context, the E-term is absorbed by the demeaning-procedure, yielding the ORU-FE specification. Again, assuming only linear effects, both $\beta_{O}$ and $\beta_{M M}$, the shared linear component of $\beta_{O Q}$ and $\beta_{U Q}$ have to be estimated from the same changes of occupation. There is hence again an identification problem. As in the cross-sectional case, the combination of both parameters is identified as $\theta_{2}=\beta_{O}-\beta_{M M}$. In order to learn something about, $\beta_{M M}$ we must make assumptions about $\beta_{O}$. Unfortunately, $\beta_{M M}$ and $\beta_{O}$ may take the same direction, so that constraining the sign of $\beta_{O}$ is usually not informative about the range of values $\beta_{M M}$ can take. Instead, we must specify a maximal effect size for $\beta_{O}$ in order to infer $\beta_{M M}$.

Since it is impossible to know such a maximal effect size a priori, I gauge the potential for causal mismatch effects by again resorting to bounded estimates. The endpoints of the bounds are comprised of the two extreme cases: that the linear effect of changing occupation is entirely due to the linear mismatch-component, and that there is no linear effect of mismatch. To the respective estimates of $\beta_{M M}$ I add the non-linear components of mismatches. Concretely, I allow different coefficients for moving deeper into overqualification, relative to actors' multi-year average, and vice versa for moving deeper into underqualification, relative to actors' multi-year average. This procedure leaves us with conservative bounds on the effects of underand overqualification. In many cases, it allows demonstrating or ruling out mismatch-effects, even when the relative size of the linear component is unclear.

\section{Results}

Is there any evidence that mismatched workers are affected by the dynamics hypothesized by SIT scholars? In the following, I report results from two sets of models: the pooled E-ORU specification and the ORU-FE model.

Cross-sectional results

Figure 3-2 shows the ranges of estimates of under- and overqualification effects that are compatible with my assumptions about the DGP and the data in the E-ORU 
specification. I refer readers to Supplement B for a comparison of these results to those obtained from a conventional ORU decomposition. Black bars indicate that all estimates are statistically significant at the 0.1 level. 

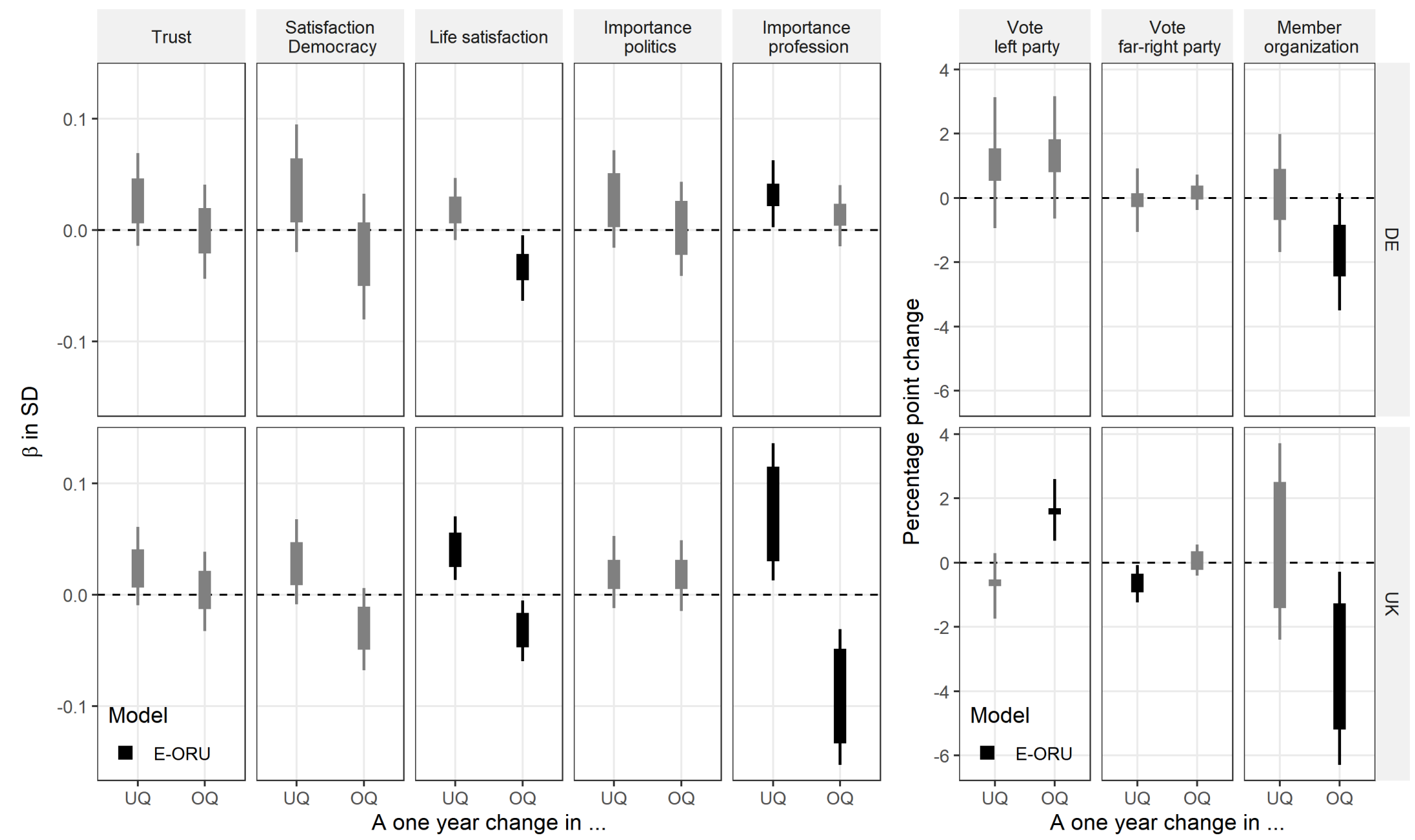

Figure 0-2: Social and political attitudes, and wages among mismatched workers

Note: Constrained least squares models estimated on pooled data. 95\% confidence intervals based on cluster-robust standard errors and ten imputations. Logged hourly wages: effects given in log-points. Results controlled for personal characteristics, 
I find that overqualification is associated with lowered life satisfaction, a lower likelihood of organizational membership, and, in the, UK with a clearly decreased salience of workers' professional identity and an increased probability to express voting intentions for a left-wing party. Underqualification, on the other hand, goes along with a heightened professional identity, and in the UK also with increased life satisfaction, and a lower likelihood to support the extreme right. The strength of these associations is often small, but arguably of substantive importance. A median effect size of about $0.03 \mathrm{SD}$ implies that somebody who is overqualified by three years, which roughly corresponds to the difference between the main educational categories, reports, for example, about a tenth of a standard deviation lower life satisfaction than someone with a similar occupation and job, who is not mismatched.

For all other variables, I cannot safely conclude effects of mismatch. Either the identification bounds or the confidence intervals overlap with zero, which means that DGPs that are compatible with my assumptions about the relative weights of $\beta_{O}$ and $\beta_{E}$ could have produced the data with a linear mismatch term of zero. I need to highlight, however, that, especially in my application, a failure to refute the null-hypothesis of no mismatch-effect does not imply support for the null hypothesis. My tests are very conservative, because prior knowledge about the true main effect of education and occupation is weak. In fact, for virtually all dependent variables, DGPs that imply non-zero mismatch effects are compatible with the data. To rule out mismatch effects in these cases, or to show their existence, stronger assumptions, or more data, are necessary.

Such stronger assumptions are available for two of our dependent variables: job satisfaction and wages. I have argued that they represent instances, where a direct effect of education can safely be assumed away. The first panel of Figure 3-3 demonstrates that overqualification is associated with lower job satisfaction, whereas underqualification goes together with higher satisfaction in the UK. This is true for a model using the assumption of zero education effects, represented by the circle marker, as well as for an E-ORU model, where I instead use the assumption of non-zero/equal-sign effects of both main-dimension. In this case, the 
returned mismatch effects are even more drastic, but possibly overstated, because they require that education per se increases job satisfaction. ${ }^{6}$
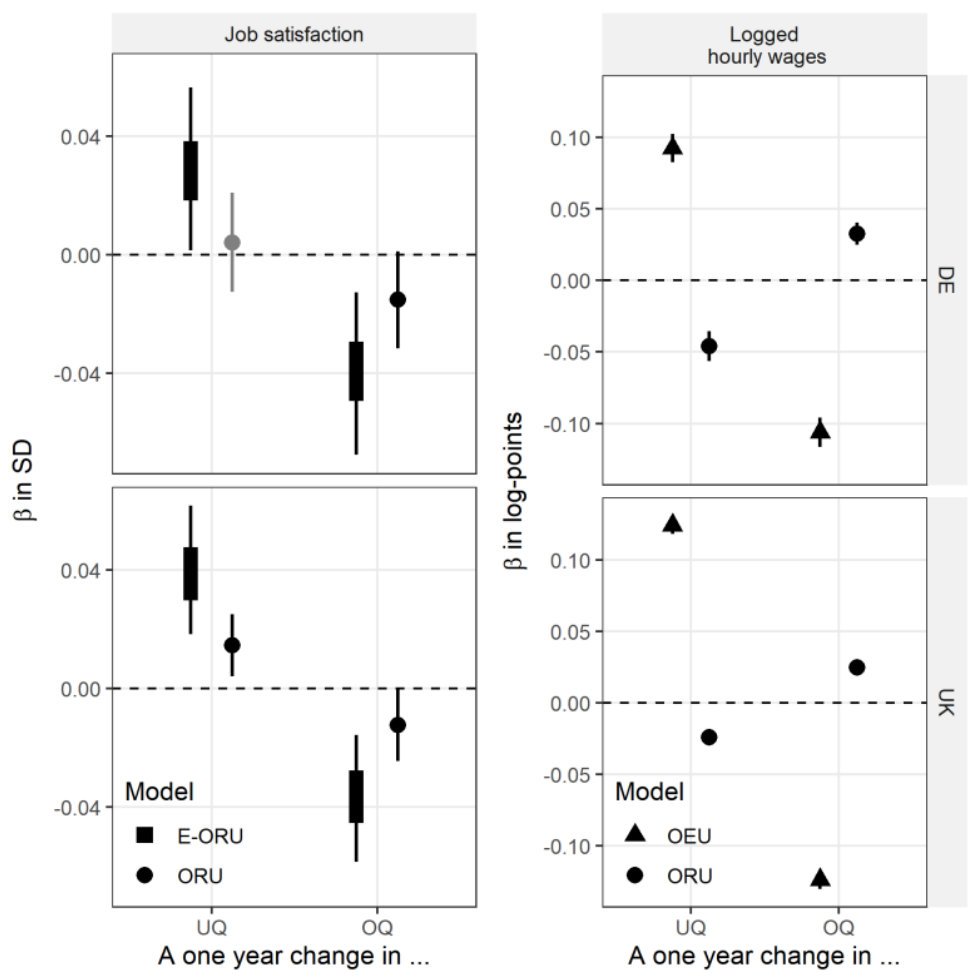

Figure 0-3. Social and political behaviors among mismatched workers

Note: Constrained linear probability models estimated on pooled data. $95 \%$ confidence intervals based on cluster-robust standard errors and ten imputations. Results controlled for personal characteristics.

What could drive the association between mismatch, satisfaction and the other relationships I have documented? Wages are an obvious candidate. But when I turn to the second panel of Figure 3-3 and thus to the results for hourly wages, the first

\footnotetext{
${ }^{6}$ One might even argue that the main effect of education should be negative, representing the idea that more schooling makes workers more demanding. I pursued this idea but did not find the evidence in its favor compelling. In a model, where the main effect of education is equal to the main effect of typical education, none of the main dimensions' effects is significantly different from zero. What is more, this model would indicate that overeducation makes people more satisfied, something that seems hard to believe.
} 
thing to note is that in both countries overqualified workers earn about 0.03 logpoints, i.e. about $3 \%$, more per hour than matched workers in the same occupation, and that underqualified workers earn less, net of all the personal characteristics I control for. This pattern is represented by the circle markers. As I have noted above, when the main effect of education is assumed to be zero in the E-ORU model, it collapses to the conventional ORU decomposition. And indeed, my results on job satisfaction and wages echo findings from that literature (Korpi and Tåhlin 2009; Vaisey 2006).

However, I can also compare mismatched to matched workers with similar education. This approach highlights the opportunity costs to mismatching and takes into account that while overqualification might result in higher wages within one occupation, wages might have been even higher had overqualified workers found matched employment. Technically, this amounts to replacing required education $(\mathrm{R})$ with actual education (E) in an ORU regression. The triangle markers provide the estimates of this OEU specification. In line with the rest of my results, I find that underqualification is associated with significant net-gains, and overqualification with large net-losses.

The first conclusion to draw from the empirical analysis is that the proposition of mismatch-effects without any linear component is not borne out by the data. This casts doubt on results obtained from the square additive model, from Zhang's model, and on Lenski's original formulation. For virtually all dependent variables, I find that over- and underqualification are associated with an outcome in opposing directions, even though my empirical model does in no way require such a pattern.

This is also an important finding for status inconsistency research on a theoretical level. While mismatches are clearly consequential for many outcomes, it does not appear to be the first and second psychological mechanisms proposed by status inconsistency theory, i.e. role conflict and cognitive dissonance, that result in dissatisfaction and withdrawal. It is not inconsistency per se that causes discontent. Rather, the negative consequences of mismatches expected by SIT are only present among the overqualified. This pattern is compatible with the third channel discussed above. It predicts opposing consequences for under- and overqualification as a result of an expectation formation mechanism: Discontent arises because prior expectations of occupational advancement, as instilled by education and training, 
have not been met. The underqualified, vice versa, report, if anything, higher levels of satisfaction. Presumably, this is because they exceed their own expectations, and hence experience the socio-economic environment as particularly fair.

My results are less clear about the fourth mechanism in SIT, which claims that mismatch-experiences in the occupational domain spill over into the domain of political attitudes and behaviors. While all indicators that pertain directly to the world of work are clearly connected to mismatches in the E-ORU model, such patterns are weaker for political variables, which hardly reach statistical significance. What seems rather robust, however, is that the overqualified are less likely to be members of organizations.

One potential point of skepticism regarding these findings arises from the fact that in the E-ORU model mismatch effects are partially identified by explicit assumptions about the relative importance of education and occupation. How sensitive are my conclusions to these assumptions? I provide results for weaker assumptions in Online Supplement D. Here I note that the core of my results, i.e. those for job and life satisfaction, the importance of one's profession, wages, leftvote and organizational membership, are substantively unaffected by the choice of identifying assumptions.

\section{Fixed-effects results}

My discussion so far has assumed that mismatches cause views and behaviours. But this need not be so. People who end up in overqualification may have been different even before they became overqualified. I test the robustness of my results in the face of such concerns using fixed-effects models that control for all time-constant heterogeneity between individuals. This is only possible, however, for variables, where repeated measurements are available.

In my models, I allow for heterogeneous mismatch effects depending on whether a worker's current value of the linear mismatch term $\left(X_{E}-X_{O}\right)$ is above (relative overqualification) or below (relative underqualification) the personspecific average across the period in the sample. 
Figure 0-4: Changes in social and political attitudes, and wages after changes of occupation
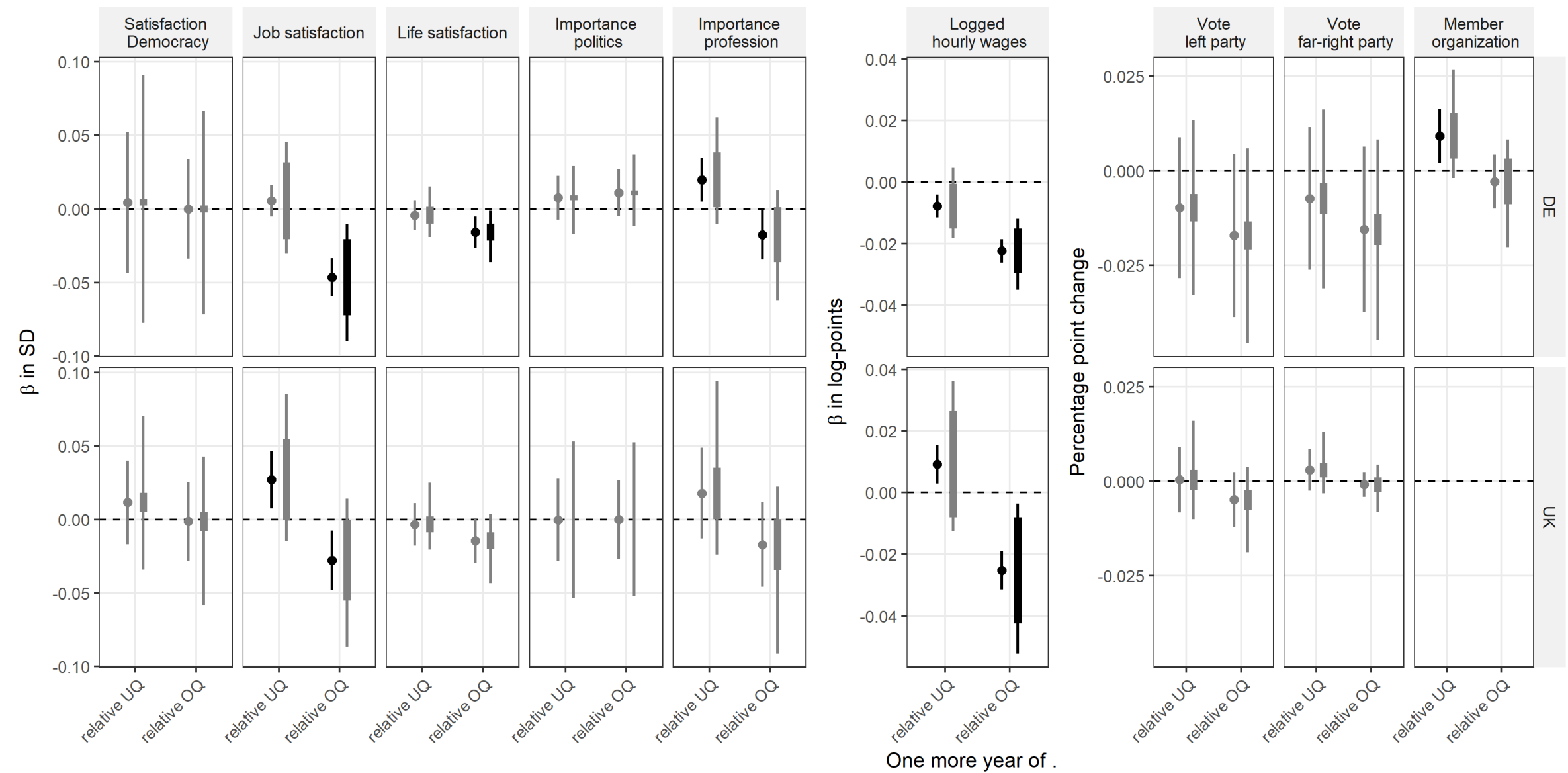

Note: Constrained least squares fixed effects models. $95 \%$ confidence intervals based on cluster-robust standard errors and ten imputations. Controls for age, tenure, and survey year. 
Figure 3-4 gives the bounded estimates of mismatch-effects (bars) and the point estimates that result from assuming equal linear effects of mismatch and of occupation (circles). As explained above, the bounded estimates represent results for assuming that between all and none of the linear portion of effects are due to the mismatch component.

The results of these very conservative tests confirm the findings from the crosssectional analysis. I find that outcomes close to the employment relationship are affected by changing mismatch-states. Relative overqualification decreases wages and satisfaction (the satisfaction variables are, however, not statistically significant in the UK). If we are willing to believe that half of the observed change in the personal importance attached to one's profession is due to the changing mismatch (as opposed to occupational) status (circle marker), we also find an effect on this outcome in Germany.

The effects of increasing relative undereducation appear to be weaker, but are present in the case of organizational membership, wages, and the importance of a professional identity in Germany - if we are willing to assume that some of the effects of job-changes are due to mismatches. Interestingly, I find that German workers, who move into relative underqualification, likely earn a bit less then implied by the occupation-change alone, whereas British workers likely earn a bit more. While my weak assumptions do not allow a definitive conclusion, this pattern seems to suggest that relative underqualification is less penalized in the UK than in Germany. Once we move towards attitudes and behaviours relating to politics, there is hardly any evidence for an effect of mismatch-changes, or, for that matter, of occupation changes, at all. Again, this echoes the results of the cross-sectional analysis. Across all outcomes, I find more statistically significant effects in the German data. This could be interpreted as a country difference. However, my longitudinal German data is much richer, because of the larger number of observations and years I can draw on. Therefore, the dissimilarities apparent in Figure 3-4 are in all likelihood the result of lower statistical power in the British sample, rather than a reflection of genuine differences between the countries. All things considered, the results of the longitudinal models thus support the conclusions from the cross-sectional analysis. 


\section{Conclusion}

Do qualification-to-job mismatches have consequences for the social and political attitudes of affected workers? This article started out with a critique of previous efforts to answer this question. I argued that the theoretical commitments of conventional strategies do not permit an answer except under very specific circumstances. Referring to the most recent age-period-cohort literature, I instead introduced a framework that uses explicit restrictions on the theoretical model based on substantive reflection to bring us closer to a solution in a wider range of scenarios.

I find that under weak and plausible assumptions, mismatched differ from matched workers beyond what is implied by their differing occupations and qualifications alone in well-being, identity, and social integration. Mismatch or inconsistency is therefore an important concept in studying the subjective experience of social stratification. Conservative fixed-effects estimators that tackle the issue of selection bias confirm the gist of my cross-sectional findings.

While I was able to show that mismatch or status inconsistency does have important consequences for the individual, my analyses nevertheless refute some of the core hypotheses of status inconsistency theory. First, I find that the most important psychological mechanism assumed by status inconsistency theory, cognitive dissonance, is unlikely to account for the observed patterns. The predicted pattern of dissatisfaction, distance from professional roles, social withdrawal, and political opposition, is evident only for the overqualified. The underqualified, however, despite their mismatch, are more satisfied, identify more with their professional role and are not more critical of the democratic system. In this sense, undereducation does not seem to be a problematic condition. All this suggests that it is not role incongruences as such, but the specific experience of underachievement that is at the root of the strains described by status inconsistency theory.

Second, while scholarly debate has strongly focused on the wider political and societal consequences of pervasive mismatch, neither the cross-sectional nor the longitudinal analyses provide convincing evidence for such a relationship. This suggests that any link between mismatch and political dissatisfaction is at most weak and likely not causal. Hence, while I was able to demonstrate that 
overqualification poses problems for the wellbeing of individuals even in a conservative within-person comparison, rising rates of mismatch are unlikely to cause widespread political alienation.

The results of my analyses are very similar between the UK and Germany. Methodologically, this builds confidence into my core results, as it demonstrates that conclusions can be replicated using a different survey study and slightly different questionnaire wording. Substantively, the evident similarity suggests that the processes I investigated take place on a rather basal psychological level and are less affected by the respective institutional context.

In the end, the substantive contributions of this study may appear somewhat paradoxical. On the one hand, the analyses have shown that classic inconsistency theory as pioneered by Lenski is hardly suited to explain the experience of mismatched workers. On the other hand, however, the main result of this study that a mismatched employment situation affects the wellbeing of individuals beyond occupation and education - provides an occasion for sociology to reinvigorate research into the multi-dimensionality of social status. The experience of falling short of institutionalized expectations, but also of exceeding them, seems to provide workers with an independent source of strain, or satisfaction, respectively.

Beyond the question of mismatches, I would like this study to be seen as an application of a broader conceptual point. As the conflation of mismatch and education effects in the ORU model demonstrates, the fact that a quantity is readily measurable does in no way mean that it corresponds to the process that actually generated the data. Vice versa, the fact that a parameter is not empirically identified does not mean that it is theoretically, or indeed in reality, meaningless. In this sense, sociological research can profit from distinguishing much more sharply between theoretical ("structural") models of the data generating process and the empirical ("reduced form") models that can in fact be statistically estimated. 


\section{Literature}

Blalock, Hubert M. 1966. 'The Identification Problem and Theory Building: The Case of Status Inconsistency'. American Sociological Review 31(1):52.

Blocker, T. Jean and Paul L. Riedesel. 1978. 'The Nonconsequences of Objective and Subjective Status Inconsistency: Requiem for a Moribund Concept'. The Sociological Quarterly 19(2):332-39.

Buck, Nick and Stephanie McFall. 2011. 'Understanding Society: Design Overview'. Longitudinal and Life Course Studies 3(1):5-17.

Burris, Val. 1983. 'The Social and Political Consequences of Overeducation'. American Sociological Review 48(4):454.

Deutsches Institut Für Wirtschaftsforschung. 2017. 'Sozio-Oekonomisches Panel (SOEP), Daten Der Jahre 1984-2016'.

Dudal, Pieter and Piet Bracke. 2019. 'On the Moderation of the Relation between Overeducation and Depressive Symptoms through Labor Market and Macro-Economic Factors'. Health \& Place 56:135-46.

Duncan, Greg J. and Saul D. Hoffman. 1981. 'The Incidence and Wage Effects of Overeducation'. Economics of Education Review 1(1):75-86.

Duncan, Otis Dudley. 2005. 'Methodological Issues in the Analysis of Social Mobility'. Pp. 51-97 in Social Structure and Mobility in Economic Development, edited by S. Lipset. New York: Routledge.

Dunlavy, A. C., A. M. Garcy, and M. Rostila. 2016. 'Educational Mismatch and Health Status among Foreign-Born Workers in Sweden'. Social Science \& Medicine 154:36-44.

Felstead, Alan, Duncan Gallie, Francis Green, Yingxiong Zhou, and Centre on Skills, Knowledge and Organisational Performance, eds. 2007. Skills at Work, 1986 to 2006. Oxford: ESRC Centre on Skills, Knowledge and Organisational Performance.

Festinger, Leon. 1962. A Theory of Cognitive Dissonance. Stanford University Press.

Fosse, Ethan and Christopher Winship. 2019a. 'Analyzing Age-Period-Cohort Data: A Review and Critique'. Annual Review of Sociology 45(1):467-92. 
Fosse, Ethan and Christopher Winship. 2019b. 'Bounding Analyses of Age-Period-Cohort Effects'. Demography.

Geschwender, James A. 1967. 'Continuities in Theories of Status Consistency and Cognitive Dissonance'. Social Forces 46(2):160-71.

Geschwender, James A. 1968. 'Status Inconsistency, Social Isolation, and Individual Unrest'. Social Forces 46(4):477-83.

Goffman, Irwin W. 1957. 'Status Consistency and Preference for Change In Power Distribution'. American Sociological Review 22(3):275.

Hendrickx, John, Nan Dirk De Graaf, Jan Lammers, and Wout Ultee. 1993. 'Models for Status Inconsistency and Mobility: A Comparison of the Approaches by Hope and Sobel with the Mainstream Square Additive Model'. Quality and Quantity 27(4):335-52.

Hope, Keith. 1975. 'Models of Status Inconsistency and Social Mobility Effects'. American Sociological Review 40(3):322-43.

Horowitz, Jonathan. 2018. 'Relative Education and the Advantage of a College Degree'. American Sociological Review 83(4):771-801.

Jackson, Elton F. 1962. 'Status Consistency and Symptoms of Stress'. American Sociological Review 27(4):469.

Jackson, Elton F. and Peter J. Burke. 1965. 'Status and Symptoms of Stress: Additive and Interaction Effects'. American Sociological Review 30(4):556-64.

Jackson, Elton F. and Richard F. Curtis. 1972. 'Effects of Vertical Mobility and Status Inconsistency: A Body of Negative Evidence'. American Sociological Review 37(6):701.

Korpi, Tomas and Michael Tåhlin. 2009. 'Educational Mismatch, Wages, and Wage Growth: Overeducation in Sweden, 1974-2000'. Labour Economics 16(2):183-93.

Lenski, Gerhard. 1964. 'Comment'. Public Opinion Quarterly 28(2):326-30.

Lenski, Gerhard E. 1954. 'Status Crystallization: A Non-Vertical Dimension of Social Status'. American Sociological Review 19(4):405-13.

Lenski, Gerhard E. 1956. 'Social Participation and Status Crystallization'. American Sociological Review 21(4):458-64. 
Levels, M., R. van der Velden, and J. Allen. 2014. 'Educational Mismatches and Skills: New Empirical Tests of Old Hypotheses'. Oxford Economic Papers 66(4):959-82.

McGuinness, Seamus. 2006. 'Overeducation in the Labour Market'. Journal of Economic Surveys 20(3):387-418.

Niemi, R. G. and B. I. Sobieszek. 1977. 'Political Socialization'. Annual Review of Sociology 3:209-33.

O’Brien, Robert M. 2014. 'Estimable Functions in Age-Period-Cohort Models: A Unified Approach'. Quality \& Quantity 48(1):457-74.

Olsen, Marvin E. and Judy Corder Tully. 1972. 'Socioeconomic-Ethnic Status Inconsistency and Preference for Political Change'. American Sociological Review 37(5):560.

Portes, Alejandro. 1972. 'Status Inconsistency and Lower-Class Leftist Radicalism'. The Sociological Quarterly 13(3):361-82.

Rohrbach-Schmidt, Daniela and Michael Tiemann. 2016. 'Educational (Mis) Match and Skill Utilization in Germany: Assessing the Role of Worker and Job Characteristics'. Journal for Labour Market Research 1-21.

Sears, David O. and Christia Brown. 2013. 'Childhood and Adult Political Development'. Pp. 59-95 in The Oxford Handbook of Political Psychology, edited by L. Huddy, D. O. Sears, and J. S. Levy. Oxford, New York: Oxford University Press.

Sloane, P. J., H. Battu, and P. T. Seaman. 1999. 'Overeducation, Undereducation and the British Labour Market'. Applied Economics 31(11):1437-53.

Smith, Brendan T., Peter M. Smith, Jacob Etches, and Cameron A. Mustard. 2012.

'Overqualification and Risk of All-Cause and Cardiovascular Mortality: Evidence From the Canadian Census Mortality Follow-up Study (1991-2001)'. Canadian Journal of Public Health 103(4):e297-302.

Sobel, Michael E. 1981. 'Diagonal Mobility Models: A Substantively Motivated Class of Designs for the Analysis of Mobility Effects'. American Sociological Review 46(6):893.

Stryker, Sheldon and Anne Statham Macke. 1978. 'Status Inconsistency and Role Conflict'. Annual Review of Sociology 4(1):57-90. 
Vaisey, Stephen. 2006. 'Education and Its Discontents: Overqualification in America, 19722002'. Social Forces 85(2):835-864.

Van Buuren, Stef. 2012. Flexible Imputation of Missing Data. CRC press.

Verba, Sidney, Norman H. Nie, and Jae-on Kim. 1978. Participation and Political Equality. New York: Cambridge University Press.

Zhang, Xiaotian. 2008. 'Status Inconsistency Revisited: An Improved Statistical Model'. European Sociological Review 24(2):155-68.

Zhu, Rong and Linfeng Chen. 2016. 'Overeducation, Overskilling and Mental Well-Being'. The B.E. Journal of Economic Analysis \& Policy 16(4). 